\title{
Analysis of bacterial diversity in the intestine of grass carp (Ctenopharyngodon idellus) based on 165 rDNA gene sequences
}

\author{
Shaofeng Han ${ }^{1}$, Yuchun Liu', Zhigang Zhou', Suxu He ${ }^{1}$, Yanan Cao $^{1}$, Pengjun Shi $^{1}$, Bin Yao ${ }^{1}$ \& \\ Einar Ringo ${ }^{2}$ \\ ${ }^{1}$ Key Laboratory for Feed Biotechnology of the Ministry of Agriculture, Feed Research Institute, Chinese Academy of \\ Agricultural Sciences, Beijing, China \\ ${ }^{2}$ Norwegian College of Fishery Science, Faculty of Biosciences, Fisheries and Economics, University of Tromsø,Tromsø, Norway
}

Correspondence: B Yao and Z Zhou, Key Laboratory for Feed Biotechnology of the Ministry of Agriculture, Feed Research Institute, Chinese Academy of Agricultural Sciences, No.12 Zhongguancun South Street, Beijing 100081, China. E-mail: yaobin@caas-bio.net.cn; zhou.zg@msn.com

\begin{abstract}
In the current study, we assessed bacterial diversity in the gut content of pond-reared grass carp (Ctenopharyngodon idellus), in the associated habitat environments (pond water and sediment) and in the ingested food (commercial feed and the reed Phragmites australis) by analysing $16 \mathrm{~S}$ rDNA sequences from clone libraries. The highest bacterial diversity was observed in the gut content and was determined by the total number of operational taxonomic units, Shannon diversity index $(H)$, Shannon equitability index $\left(E_{H}\right)$, Coverage $\left(C_{\text {good }}\right)$ and rarefaction curves calculated from the $16 \mathrm{~S}$ rDNA gene libraries. Our data indicated that allochthonous gut microbes of grass carp were distinctively different from the corresponding environmental microbes. The pairwise similarity coefficient $\left(C_{\mathrm{s}}\right)$ for microbe communities between gut content and ingested food was higher than for those between the gut content and habitats, indicating that the allochthonous microbiota identified in the intestines of grass carp were phylogenetically closer to those in the ingested food than to those in the habitat. Based on our study and previous research, we suggest that the digesta of grass carp harbours a microbiota phylogenetic core of Proteobacteria and Firmicutes and this observation deserves further investigations with respect to a potential pool of probiotics to grass carp.
\end{abstract}

Keywords: grass carp, Ctenopharyngodon idellus, intestinal bacterial diversity, 16S rDNA

\section{Introduction}

Gastrointestinal (GI) microbiota participate in several important physiological functions of the host, including digestion, development of the mucosal system, angiogenesis and protection against disease (Macfarlane \& Macfarlane 1997; Hooper, Midtvedt \& Gordon 2002). It is generally accepted that identification of the GI microbiota is undoubtedly important for understanding the functional mechanisms between the microbes and the host (Gómez \& Balcázar 2008). Difficulties in analysing the complexity of bacterial community using classic methods of cultivation have necessitated the development of molecular methods. In order to overcome these problems, various methods such as denaturing gradient gel electrophoresis (DGGE) (Muyzer, Waal \& Uitterlinden 1993), fluorescence in situ hybridization (Huber, Spanggaard, Appel, Rossen, Nielsen \& Gram 2004), temporal temperature-gradient electrophoresis (Navarrete, Magne, Mardones, Riveros, Opazo, Suau, Pochart \& Romero 2010) and clone libraries (Kim, Brunt \& Austin 2007; Brons \& Elsas 2008; Navarrete, Espejo \& Romero 2009; Ward, Blaire, Penn, Methé \& Detrich 2009) have been used in order to circumvent the need for microbial isolation.

The DGGE-based method is a useful tool for separating gene fragments but has strict length limitations (generally < 500 bp) (Myers, Fischer, Lerman \& Maniatis 1985) and often fails to establish an exact identification of the fragments using the BLAST program (Altschul, Gish, Miller, Myers \& Lipman 1990). In 
addition, it only detects the dominant bacterial species in the environments (Muyzer et al. 1993). The generation of $16 \mathrm{~S}$ rDNA clone libraries that contain near-full-length 16S rDNA sequences would likely result in more precise sequence identification than sequences obtained from DGGE (Brons \& Elsas 2008).

The grass carp (Ctenopharyngodon idellus) is a herbivorous freshwater fish of the Cyprinidae family and these fish are widely cultivated for food in China. The output was $>4$ million tonnes in 2008 and comprised $>20 \%$ of the total freshwater-cultured fish annual output (Ministry of Agriculture, China 2009). Members of the Cyprinidae family have also been introduced to Europe and the United States for aquatic weed control (Chilton \& Muoneke 1983).

During the last three decades, some papers have been published in which the gut microbiota of grass carp were identified using traditional methods such as freshwater agar and some selective culture media (Trust, Bull, Currie \& Buckley 1979; Zhou, Chen, Zhang \& Chen 1998; Luo, Chen \& Cai 2001; He, Zhang, Xie, Hao, Wang \& He 2008). Recently, Huang, Shi, Wang, Luo, Shao, Wang, Yang and Yao (2009) studied the intestinal bacterial community of grass carp by PCR amplification of the $\mathrm{V} 3$ region of $16 \mathrm{~S}$ rDNA and by DGGE; to our knowledge, a $16 \mathrm{~S}$ rDNA clone library has not been generated for the identification of grass carp gut microbiota, however.

The diversity of the GI microbiota of fish is influenced by environmental factors such as ingested food and habitat (Sugita, Oshima, Tamura \& Deguchi 1983; Nieto, Toranzo \& Barja 1984). However, the correlation between gut microbiota and its corresponding environmental microbiota is per se not fully understood, and whether the grass carp gut harbours a microbiota phylogenetic core (the common phyla within the gut contents of grass carp from different backgrounds) has not been addressed. In the present study, we identified the allochthonous intestinal microbiota of the grass carp by generating a 16S rDNA library comprised of sequences from samples of grass carp gut content, the associated habitat (pond water and sediment) and the ingested food (commercial feed and natural food).

\section{Materials and methods}

\section{Sample preparation}

Grass carp was raised in a poly-culture pond of grass carp, gibel carp (Carassius auratus gibelio) and blunt- nose black bream (Megalobrama amblycephala) in the suburb of Nanjing City, Jiangsu Province, China. A description of the pond as well as the fish and pond water, sampling methods and sampling procedure is presented in Wang, Zhou, He, Liu, Cao, Shi, Yao and Ringø (2010). Twelve grass carp were randomly collected from the pond. Pond water and sediment samples were collected from the same pond locations $(n=4)$. Sediment samples were collected using a mud dredger (VG, Beijing Purity Instruments, Beijing, China) and were pooled before analysis. In addition, feed samples $(\sim 200 \mathrm{~g})$ were obtained from the automatic feeder. Samples of the reed (Phragmites australis) available for ingestion by grass carp were collected from four randomly chosen sites in the pond. Samples were stored on ice for transport to the lab and then kept at $-20{ }^{\circ} \mathrm{C}$ until analysis. After examining all fish (12), gut contents from six grass carp having identical gut fullness were used. The gut samples chosen were visually full of food ingested and the digesta were gently squeezed out under sterile conditions and pooled before analysis. Pooled samples were used to avoid erroneous conclusions due to individual variations in gut microbiota as described elsewhere (Spanggaard, Huber, Nielsen, Nielsen, Appel \& Gram 2000; He, Zhou, Liu, Shi, Yao, Ringø \& Yoon 2009).

\section{DNA extraction}

Total DNA was extracted from $5 \mathrm{~g}$ sediment or feed as described by Tsai and Olson (1991). Extracted DNA was purified using the Gel Cycle-Pure DNA kit (Takara, Tokyo, Japan) according to the manufacturer's instructions and used as template DNA for PCR amplification. DNA was extracted from pond water as described elsewhere (Gernert, Glöckner, Krohne \& Hentschel 2005). DNA was extracted from fish gut content using the hexadecyltrimethylammonium bromide (CTAB) method, which involves a step of suspending the samples in CTAB extraction buffer (Thakuria, Schmidt, Mac Siúrtáin, Egan \& Doohan 2008). To obtain reed DNA, 5 g reed sample was cut into small pieces, transferred to a sterile triangular flask containing $20 \mathrm{~mL}$ PBS buffer and 10 glass beads $(0.5 \mathrm{~cm}$ diameter) and then agitated at $4 \mathrm{~g}$ for $30 \mathrm{~min}$. The mixture was allowed to settle for $10 \mathrm{~min}$, and the supernatant was transferred into a sterile tube and centrifuged at $14000 \times g$ for $15 \mathrm{~min}$ at $4{ }^{\circ} \mathrm{C}$. Total DNA was extracted from the precipitate using the DNA extraction kit (Takara). 


\section{PCR amplification and $16 S$ rDNA library construction}

Universal primers 27f and 1492r (Martin-Laurent, Philippot, Hallet, Chaussod, Germon, Soulas \& Catroux 2001), which anneal at nucleotide positions 8-27 and 1492-1513 of the 16S rDNA gene (Escherichia coli numbering), respectively, were used for $16 \mathrm{~S}$ rDNA library construction. PCR reaction conditions were as described by Martin-Laurent et al. (2001). PCR products ( $\sim 1300 \mathrm{bp}$ ) were purified, cloned into the pGEM-T vector and transformed into E. coli XL1blue (Promega, Southampton, UK) according to the manufacturer's instructions. Blue/white selection was used for clone screening. For each sample type, $\sim 100$ clones containing correct inserts ( $\sim 1300 \mathrm{bp}$ ) were randomly selected, verified by PCR amplification using the 27f/1492r primer set and sequenced by Sunbiotech (Beijing, China).

\section{Data analysis}

The 16S rDNA clone library sequences were submitted to the CHECK.CHIMERA program of the Ribosomal Database Project to detect possible chimeric artefacts (Cole, Chai, Farris, Wang, Julam, McGarrel, Garrity \& Tiedje 2005). All sequences were subjected to similarity searches using the BLAST program (Altschul et al. 1990) after removing unreliable sequences at the $3^{\prime}$ and $5^{\prime}$ ends.

Using the TSYS-PC program (version 2.1, Jandel Scientific, San Rafael, CA, USA), sequences identified in the current study were integrated into an annotated tree based on parsimony. The relative abundance (\%) of an operational taxonomic unit (OTU; the clones with $100 \%$ sequence similarity), representing the ratio of the number of the clones of a specific OTU to the total number of clones, was considered to be significant when the value was more than 1.5-fold higher or less than 0.5-fold lower than the abundance of any other OTU. The Shannon diversity index was calculated using the equation $H=-\Sigma R A_{i}$ $\ln \left(R A_{i}\right)$, and the Shannon equitability index was calculated using the equation $E_{H}=H / \ln (S)$ (where $R A_{i}$ is the proportion of the ith OTU and $S$ is the total number of OTUs) (Dethlefsen, Huse, Sogin \& Relman 2008). The Coverage $\left(C_{\text {good }}\right)$ was calculated according to Good (1953) using the equation $C_{\text {good }}=1-N_{1} /$ the total number of OTUs (where $N_{1}$ is the number of OTUs with only one clone). Cluster analysis was based on the unweighted pair group method using the arithmetic mean algorithm (UPGMA). In this study, the bacterial communities with a pairwise similarity coefficient $\left(C_{\mathrm{S}}\right.$ : the measure of the similarity of two samples by UPGMA) $<0.60$ were regarded as different, those with $0.60 \leq C_{\mathrm{s}}<0.80$ were considered to be marginally different and those with $C_{\mathrm{s}} \geq 0.80$ were considered to be similar (Wang et al. 2010). Rarefaction curves were created using the species diversity function of the ECOSIM 700 statistical software (Gotelli \& Entsminger 2002).

\section{Results}

The phylogenetic affiliations of the $16 \mathrm{~S}$ rDNA genes isolated from the gut content of grass carp and from corresponding habitat and food samples are shown in Table 1. After removing unreliable sequence data, a total of 490 clones were identified, including 100 clones from gut samples, 102 clones from feed samples, 88 clones from reed samples, 100 clones from pond water samples and 100 clones from sediment samples. The dominant bacterial phylum identified in each sample type was Proteobacteria (Fig. 1). Specifically, the dominant class of bacteria in grass carp gut, feed, pond water and sediment samples was $\gamma$-Proteobacteria, which comprised $28.0 \%, 33.3 \%$, $46.0 \%$ and $49.0 \%$, respectively, of the total bacterial content. The dominant class in reed samples was Bacteroidetes, with a relative abundance of $18.2 \%$ (Table 1). However, unclassified bacteria comprised a large proportion of the bacteria in each sample type: $21.0 \%, 31.4 \%, 13.6 \%, 42.0 \%$ and $37.0 \%$ in gut content, feed, reed, pond water and sediment samples respectively. The OTUs with the greatest relative abundance in gut content, feed, reed, pond water and sediment were OTU36 (99\% similarity to Pseudomonas aeruginosa; FM209186), OTU10 (99\% similarity to Bacillus sp.; AY822760), OTU34 (98\% similarity to uncultured $\beta$-Proteobacterium; EU753670), OTU64 (99\% similarity to the uncultured bacterium, DQ394301) and OTU35 (100\% similarity to Enterobacter sp.; EF175731) respectively (Table 1).

The diversity of the allochthonous intestinal bacterial community in the grass carp gut and in the corresponding ecosystem components is presented in Fig. 2. The total number of OTUs was the highest in the gut content (48), followed by pond water (29), reed (19), sediment (18) and feed (13). The Shannon diversity index $(H)$ in the gut content was 3.465 , higher than that in the associated habitat and food samples. Similar trends were observed in the Shannon equitability index $\left(E_{H}\right)$ values and the Coverage 
Table 1 Phylogenetic affiliation of 16S rDNA gene phylotypes isolated from the gut content of grass carp and from associated food and habitat samples*

\begin{tabular}{|c|c|c|c|c|c|c|c|c|}
\hline \multirow[b]{2}{*}{ оTU } & \multicolumn{5}{|c|}{ Relative abundance (\%) } & \multirow[b]{2}{*}{$\begin{array}{l}\text { Closest relative in GenBank } \\
\text { (accession no.) }\end{array}$} & \multirow[b]{2}{*}{$\begin{array}{l}\text { Similarity to } \\
\text { the closest } \\
\text { relative }(\%)\end{array}$} & \multirow[b]{2}{*}{$\begin{array}{l}\text { Phylogenetic } \\
\text { group }\end{array}$} \\
\hline & $\begin{array}{l}\text { Gut content } \\
\text { (100 clones) }\end{array}$ & $\begin{array}{l}\text { Feed } \\
\text { (102 } \\
\text { clones) }\end{array}$ & $\begin{array}{l}\text { Reed } \\
\text { (88 } \\
\text { clones) }\end{array}$ & $\begin{array}{l}\text { Water } \\
\text { (100 } \\
\text { clones) }\end{array}$ & $\begin{array}{l}\text { Sediment } \\
\text { (100 } \\
\text { clones) }\end{array}$ & & & \\
\hline OTU1 & $1.0^{\mathrm{b}}$ & $0.0^{\mathrm{a}}$ & $0.0^{\mathrm{a}}$ & $0.0^{\mathrm{a}}$ & $0.0^{\mathrm{a}}$ & Actinomyces naes/undii (AJ635359.1) & 94 & Actinobacteridae \\
\hline OTU2 & $2.0^{\mathrm{b}}$ & $0.0^{\mathrm{a}}$ & $1.1^{\mathrm{b}}$ & $0.0^{\mathrm{a}}$ & $0.0^{\mathrm{a}}$ & Arthrobacter sp. (AJ810894.1) & 98 & Actinobacteridae \\
\hline OTU3 & $1.0^{\mathrm{b}}$ & $0.0^{\mathrm{a}}$ & $0.0^{\mathrm{a}}$ & $0.0^{\mathrm{a}}$ & $0.0^{\mathrm{a}}$ & Curtobacterium sp. (EF411134.1) & 99 & Actinobacteridae \\
\hline OTU4 & $1.0^{\mathrm{b}}$ & $0.0^{\mathrm{a}}$ & $0.0^{\mathrm{a}}$ & $0.0^{\mathrm{a}}$ & $0.0^{\mathrm{a}}$ & $\begin{array}{l}\text { Curtobacterium flaccumfaciens } \\
\text { (AM410688.1) }\end{array}$ & 99 & Actinobacteridae \\
\hline OTU5 & $2.0^{\mathrm{b}}$ & $0.0^{\mathrm{a}}$ & $0.0^{\mathrm{a}}$ & $0.0^{\mathrm{a}}$ & $0.0^{\mathrm{a}}$ & $\begin{array}{l}\text { Microbacterium phyllosphaerae } \\
\text { (EF143430.1) }\end{array}$ & 98 & Actinobacteridae \\
\hline OTU6 & $2.0^{\mathrm{b}}$ & $0.0^{\mathrm{a}}$ & $0.0^{\mathrm{a}}$ & $0.0^{\mathrm{a}}$ & $0.0^{\mathrm{a}}$ & Bacillus coagulans (DQ297928.1) & 99 & Bacillales \\
\hline OTU7 & $1.0^{\mathrm{b}}$ & $2.0^{\mathrm{b}}$ & $0.0^{\mathrm{a}}$ & $0.0^{\mathrm{a}}$ & $0.0^{\mathrm{a}}$ & Bacillus massiliensis (DQ350816.1) & 99 & Bacillales \\
\hline OTU8 & $4.0^{c}$ & $1.0^{\mathrm{b}}$ & $0.0^{\mathrm{a}}$ & $0.0^{\mathrm{a}}$ & $0.0^{\mathrm{a}}$ & Bacillus megaterium (DQ660362.1) & 99 & Bacillales \\
\hline OTU9 & $1.0^{\mathrm{b}}$ & $0.0^{\mathrm{a}}$ & $0.0^{\mathrm{a}}$ & $0.0^{\mathrm{a}}$ & $0.0^{\mathrm{a}}$ & Bacillus pumilus (EU221329.1) & 99 & Bacillales \\
\hline OTU10 & $2.0^{\mathrm{ab}}$ & $19.6^{\mathrm{C}}$ & $4.5^{\mathrm{b}}$ & $1.0^{\mathrm{a}}$ & $2.0^{\mathrm{ab}}$ & Bacillus sp. (AY822760.1) & 99 & Bacillales \\
\hline OTU11 & $1.0^{\mathrm{b}}$ & $0.0^{\mathrm{a}}$ & $0.0^{\mathrm{a}}$ & $0.0^{\mathrm{a}}$ & $0.0^{\mathrm{a}}$ & Exiguobacterium sp. (DQ019168.1) & 99 & Bacillales \\
\hline OTU12 & $2.0^{\mathrm{b}}$ & $0.0^{\mathrm{a}}$ & $0.0^{\mathrm{a}}$ & $0.0^{\mathrm{a}}$ & $0.0^{\mathrm{a}}$ & Geobacillus toebi (AY608982.1) & 99 & Bacillales \\
\hline OTU13 & $1.0^{\mathrm{b}}$ & $0.0^{\mathrm{a}}$ & $0.0^{\mathrm{a}}$ & $0.0^{\mathrm{a}}$ & $0.0^{\mathrm{a}}$ & Staphylococcus kloosii (DQ093351.1) & 91 & Bacillales \\
\hline OTU14 & $1.0^{\mathrm{b}}$ & $0.0^{\mathrm{a}}$ & $0.0^{\mathrm{a}}$ & $0.0^{\mathrm{a}}$ & $0.0^{\mathrm{a}}$ & Ureibacillus koreensis (DQ348072.1) & 99 & Bacillales \\
\hline OTU15 & $2.0^{\mathrm{b}}$ & $0.0^{\mathrm{a}}$ & $13.6^{c}$ & $0.0^{\mathrm{a}}$ & $0.0^{\mathrm{a}}$ & $\begin{array}{l}\text { Ureibacillus thermosphaericus } \\
\text { (AB101594.1) }\end{array}$ & 99 & Bacillales \\
\hline OTU16 & $1.0^{\mathrm{b}}$ & $0.0^{\mathrm{a}}$ & $18.2 \mathrm{~d}$ & $3.0^{\mathrm{C}}$ & $0.0^{\mathrm{a}}$ & Uncultured Bacteroidetes (EF612369.1) & 94 & Bacteroidetes \\
\hline OTU17 & $1.0^{\mathrm{b}}$ & $0.0^{\mathrm{a}}$ & $0.0^{\mathrm{a}}$ & $0.0^{\mathrm{a}}$ & $0.0^{\mathrm{a}}$ & Clostridium sp. (AY188850.1) & 99 & Clostridia \\
\hline OTU18 & $1.0^{\mathrm{b}}$ & $0.0^{\mathrm{a}}$ & $0.0^{\mathrm{a}}$ & $0.0^{\mathrm{a}}$ & $0.0^{\mathrm{a}}$ & $\begin{array}{l}\text { Low } G+C \text { Gram-positive bacterium M54 } \\
\text { (AB116132.1) }\end{array}$ & 99 & Firmicutes \\
\hline OTU19 & $1.0^{\mathrm{b}}$ & $2.0^{\mathrm{b}}$ & $0.0^{\mathrm{a}}$ & $0.0^{\mathrm{a}}$ & $0.0^{\mathrm{a}}$ & Lactobacillus curvatus (EU855223.1) & 99 & Lactobacillales \\
\hline OTU20 & $1.0^{\mathrm{b}}$ & $1.0^{\mathrm{b}}$ & $2.3^{\mathrm{b}}$ & $0.0^{\mathrm{a}}$ & $0.0^{\mathrm{a}}$ & Lactobacillus fermentum (AB362626.1) & 99 & Lactobacillales \\
\hline OTU21 & $2.0^{\mathrm{b}}$ & $0.0^{\mathrm{a}}$ & $0.0^{\mathrm{a}}$ & $0.0^{\mathrm{a}}$ & $0.0^{\mathrm{a}}$ & Lactococcus lactis (AB008215.1) & 99 & Lactobacillales \\
\hline OTU22 & $7.0^{\mathrm{c}}$ & $2.9^{\mathrm{bc}}$ & $1.1^{\mathrm{b}}$ & $0.0^{\mathrm{a}}$ & $0.0^{\mathrm{a}}$ & Leuconostoc citreum (AB362721.1) & 99 & Lactobacillales \\
\hline OTU23 & $1.0^{\mathrm{b}}$ & $0.0^{\mathrm{a}}$ & $0.0^{\mathrm{a}}$ & $0.0^{\mathrm{a}}$ & $0.0^{\mathrm{a}}$ & Streptococcus (AY232833.1) & 94 & Lactobacillales \\
\hline OTU24 & $2.0^{\mathrm{b}}$ & $0.0^{\mathrm{a}}$ & $0.0^{\mathrm{a}}$ & $0.0^{\mathrm{a}}$ & $0.0^{\mathrm{a}}$ & $\begin{array}{l}\text { Streptococcus constellatus } \\
\text { (AF104676.1) }\end{array}$ & 94 & Lactobacillales \\
\hline OTU25 & $2.0^{\mathrm{bc}}$ & $0.0^{\mathrm{a}}$ & $1.1^{\mathrm{b}}$ & $4.0^{\mathrm{c}}$ & $0.0^{\mathrm{a}}$ & Streptococcus iniae (AF335572.1) & 99 & Lactobacillales \\
\hline OTU26 & $1.0^{\mathrm{b}}$ & $0.0^{\mathrm{a}}$ & $3.4^{\mathrm{c}}$ & $0.0^{\mathrm{a}}$ & $0.0^{\mathrm{a}}$ & Streptococcus parauberis (FJ009631.1) & 99 & Lactobacillales \\
\hline OTU27 & $0.0^{\mathrm{a}}$ & $4.9^{\mathrm{b}}$ & $0.0^{\mathrm{a}}$ & $0.0^{\mathrm{a}}$ & $0.0^{\mathrm{a}}$ & Streptococcus salivarius (AM157419.1) & 93 & Lactobacillales \\
\hline OTU28 & $1.0^{\mathrm{b}}$ & $2.0^{\mathrm{b}}$ & $0.0^{\mathrm{a}}$ & $0.0^{\mathrm{a}}$ & $0.0^{\mathrm{a}}$ & Wiessella confuse (DQ321751.1) & 99 & Lactobacillales \\
\hline OTU29 & $1.0^{\mathrm{b}}$ & $0.0^{\mathrm{a}}$ & $0.0^{\mathrm{a}}$ & $0.0^{\mathrm{a}}$ & $0.0^{\mathrm{a}}$ & Afipia geno sp. (U87773.1) & 99 & $\alpha$-Proteobacteria \\
\hline OTU30 & $1.0^{\mathrm{b}}$ & $0.0^{\mathrm{a}}$ & $0.0^{\mathrm{a}}$ & $0.0^{\mathrm{a}}$ & $3.0^{\mathrm{c}}$ & $\begin{array}{l}\text { Achromobacter xylosoxidans } \\
\text { (EU373389.1) }\end{array}$ & 99 & $\beta$-Proteobacteria \\
\hline OTU31 & $1.0^{\mathrm{b}}$ & $0.0^{\mathrm{a}}$ & $0.0^{\mathrm{a}}$ & $0.0^{\mathrm{a}}$ & $0.0^{\mathrm{a}}$ & Methylophilus leisingeri (AB193725.1) & 99 & $\beta$-Proteobacteria \\
\hline OTU32 & $0.0^{\mathrm{a}}$ & $0.0^{\mathrm{a}}$ & $0.0^{\mathrm{a}}$ & $0.0^{\mathrm{a}}$ & $6.0^{\mathrm{b}}$ & $\begin{array}{l}\text { Uncultured } \beta \text {-Proteobacterium } \\
\text { (FM253602.1) }\end{array}$ & 98 & $\beta$-Proteobacteria \\
\hline OTU33 & $0.0^{\mathrm{a}}$ & $0.0^{\mathrm{a}}$ & $0.0^{\mathrm{a}}$ & $0.0^{\mathrm{a}}$ & $3.0^{\mathrm{b}}$ & $\begin{array}{l}\text { Uncultured } \beta \text {-Proteobacterium } \\
\text { (EF612408.1) }\end{array}$ & 98 & $\beta$-Proteobacteria \\
\hline OTU34 & $3.0^{\mathrm{b}}$ & $0.0^{\mathrm{a}}$ & $25.0^{\mathrm{c}}$ & $4.0^{\mathrm{b}}$ & $0.0^{\mathrm{a}}$ & $\begin{array}{l}\text { Uncultured } \beta \text {-Proteobacterium } \\
\text { (EU753670.1) }\end{array}$ & 98 & $\beta$-Proteobacteria \\
\hline OTU35 & $3.0^{\mathrm{a}}$ & $15.7^{\mathrm{bc}}$ & $5.7^{\mathrm{ab}}$ & $2.0^{\mathrm{a}}$ & $22.0^{\mathrm{C}}$ & Enterobacter sp. (EF175731.1) & 100 & $\gamma$-Proteobacteria \\
\hline OTU36 & $17.0 \mathrm{~d}$ & $7.8^{\mathrm{c}} \mathrm{d}$ & $2.3^{\mathrm{ab}}$ & $1.0^{\mathrm{a}}$ & $4.0^{\mathrm{bc}}$ & $\begin{array}{l}\text { Pseudomonas aeruginosa } \\
\text { (FM209186.1) }\end{array}$ & 99 & $\gamma$-Proteobacteria \\
\hline OTU37 & $2.0^{\mathrm{bc}}$ & $9.8 \mathrm{~d}$ & $0.0^{\mathrm{a}}$ & $1.0^{\mathrm{b}}$ & $3.0^{\mathrm{c}}$ & Pseudomonas putida (CP000926.1) & 99 & $\gamma$-Proteobacteria \\
\hline OTU38 & $1.0^{\mathrm{b}}$ & $0.0^{\mathrm{a}}$ & $0.0^{\mathrm{a}}$ & $0.0^{\mathrm{a}}$ & $2.0^{\mathrm{b}}$ & Serratia liquefaciens (DQ123840.1) & 99 & $\gamma$-Proteobacteria \\
\hline OTU39 & $3.0^{\mathrm{b}}$ & $0.0^{\mathrm{a}}$ & $2.3^{\mathrm{b}}$ & $0.0^{\mathrm{a}}$ & $13.0^{\mathrm{c}}$ & Shigella sonnei (EU723822.1) & 99 & $\gamma$-Proteobacteria \\
\hline OTU40 & $0.0^{\mathrm{a}}$ & $0.0^{\mathrm{a}}$ & $0.0^{\mathrm{a}}$ & $1.0^{\mathrm{b}}$ & $0.0^{\mathrm{a}}$ & $\begin{array}{l}\text { Uncultured } \gamma \text {-Proteobacterium } \\
\text { (AF324537.1) }\end{array}$ & 99 & $\gamma$-Proteobacteria \\
\hline OTU41 & $0.0^{\mathrm{a}}$ & $0.0^{\mathrm{a}}$ & $1.1^{\mathrm{b}}$ & $4.0^{\mathrm{c}}$ & $0.0^{\mathrm{a}}$ & $\begin{array}{l}\text { Uncultured } \gamma \text {-Proteobacterium } \\
\text { (EU394575.1) }\end{array}$ & 99 & $\gamma$-Proteobacteria \\
\hline
\end{tabular}


Table 1 Continued

\begin{tabular}{|c|c|c|c|c|c|c|c|c|}
\hline \multirow[b]{2}{*}{ OTU } & \multicolumn{5}{|c|}{ Relative abundance (\%) } & \multirow[b]{2}{*}{$\begin{array}{l}\text { Closest relative in GenBank } \\
\text { (accession no.) }\end{array}$} & \multirow[b]{2}{*}{$\begin{array}{l}\text { Similarity to } \\
\text { the closest } \\
\text { relative (\%) }\end{array}$} & \multirow[b]{2}{*}{$\begin{array}{l}\text { Phylogenetic } \\
\text { group }\end{array}$} \\
\hline & $\begin{array}{l}\text { Gut content } \\
\text { (100 clones) }\end{array}$ & $\begin{array}{l}\text { Feed } \\
\text { (102 } \\
\text { clones) }\end{array}$ & $\begin{array}{l}\text { Reed } \\
\text { (88 } \\
\text { clones) }\end{array}$ & $\begin{array}{l}\text { Water } \\
\text { (100 } \\
\text { clones) }\end{array}$ & $\begin{array}{l}\text { Sediment } \\
(100 \\
\text { clones) }\end{array}$ & & & \\
\hline OTU42 & $0.0^{\mathrm{a}}$ & $0.0^{\mathrm{a}}$ & $2.3^{\mathrm{b}}$ & $6.0^{\mathrm{b}}$ & $0.0^{\mathrm{a}}$ & $\begin{array}{l}\text { Uncultured } \gamma \text {-Proteobacterium } \\
\text { (EU394575.1) }\end{array}$ & 99 & $\gamma$-Proteobacteria \\
\hline OTU43 & $0.0^{\mathrm{a}}$ & $0.0^{\mathrm{a}}$ & $0.0^{\mathrm{a}}$ & $0.0^{\mathrm{a}}$ & $2.0^{\mathrm{b}}$ & Uncultured Shigella (FJ193063.1) & 100 & $\gamma$-Proteobacteria \\
\hline OTU44 & $1.0^{\mathrm{b}}$ & $0.0^{\mathrm{a}}$ & $0.0^{\mathrm{a}}$ & $10.0^{c}$ & $0.0^{\mathrm{a}}$ & Uncultured Acinetobacter (FJ192439.1) & 99 & $\gamma$-Proteobacteria \\
\hline OTU45 & $0.0^{\mathrm{a}}$ & $0.0^{\mathrm{a}}$ & $0.0^{\mathrm{a}}$ & $1.0^{\mathrm{b}}$ & $0.0^{\mathrm{a}}$ & Uncultured Acinetobacter (FJ192480.1) & 99 & $\gamma$-Proteobacteria \\
\hline OTU46 & $1.0^{\mathrm{b}}$ & $0.0^{\mathrm{a}}$ & $0.0^{\mathrm{a}}$ & $12.0^{\mathrm{b}}$ & $0.0^{\mathrm{a}}$ & Uncultured Acinetobacter (FJ192980.1) & 100 & $\gamma$-Proteobacteria \\
\hline OTU47 & $0.0^{\mathrm{a}}$ & $0.0^{\mathrm{a}}$ & $0.0^{\mathrm{a}}$ & $1.0^{\mathrm{b}}$ & $0.0^{\mathrm{a}}$ & Uncultured Acinetobacter (FJ192631.1) & 99 & $\gamma$-Proteobacteria \\
\hline OTU48 & $0.0^{\mathrm{a}}$ & $0.0^{\mathrm{a}}$ & $0.0^{\mathrm{a}}$ & $2.0^{\mathrm{b}}$ & $0.0^{\mathrm{a}}$ & Uncultured Acinetobacter (EU407207.1) & 99 & $\gamma$-Proteobacteria \\
\hline OTU49 & $0.0^{\mathrm{a}}$ & $0.0^{\mathrm{a}}$ & $0.0^{\mathrm{a}}$ & $1.0^{\mathrm{b}}$ & $0.0^{\mathrm{a}}$ & Uncultured Acinetobacter (AF467299.1) & 99 & $\gamma$-Proteobacteria \\
\hline OTU50 & $0.0^{\mathrm{a}}$ & $0.0^{\mathrm{a}}$ & $0.0^{\mathrm{a}}$ & $1.0^{\mathrm{b}}$ & $0.0^{\mathrm{a}}$ & $\begin{array}{l}\text { Acinetobacter calcoaceticus } \\
\text { (AM157426.1) }\end{array}$ & 97 & $\gamma$-Proteobacteria \\
\hline OTU51 & $0.0^{\mathrm{a}}$ & $0.0^{\mathrm{a}}$ & $0.0^{\mathrm{a}}$ & $2.0^{\mathrm{b}}$ & $0.0^{\mathrm{a}}$ & Acinetobacter johnsonii (DQ911549.1) & 99 & $\gamma$-Proteobacteria \\
\hline OTU52 & $0.0^{\mathrm{a}}$ & $0.0^{\mathrm{a}}$ & $2.3^{\mathrm{bc}}$ & $1.0^{\mathrm{b}}$ & $3.0^{\mathrm{c}}$ & Acinetobacter sp. (EU703817.1) & 99 & $\gamma$-Proteobacteria \\
\hline OTU53 & $0.0^{\mathrm{a}}$ & $0.0^{\mathrm{a}}$ & $0.0^{\mathrm{a}}$ & $0.0^{\mathrm{a}}$ & $7.0^{\mathrm{b}}$ & Uncultured bacterium (AJ487021.1) & 99 & Unclassified \\
\hline OTU54 & $0.0^{\mathrm{a}}$ & $0.0^{\mathrm{a}}$ & $0.0^{\mathrm{a}}$ & $0.0^{\mathrm{a}}$ & $15.0^{\mathrm{b}}$ & Uncultured bacterium (AM697120.1) & 98 & Unclassified \\
\hline OTU55 & $0.0^{\mathrm{a}}$ & $0.0^{\mathrm{a}}$ & $0.0^{\mathrm{a}}$ & $0.0^{\mathrm{a}}$ & $5.0^{\mathrm{b}}$ & Uncultured bacterium (AM745142.1) & 89 & Unclassified \\
\hline OTU56 & $0.0^{\mathrm{a}}$ & $0.0^{\mathrm{a}}$ & $0.0^{\mathrm{a}}$ & $1.0^{\mathrm{b}}$ & $0.0^{\mathrm{a}}$ & Uncultured bacterium (AY661997.1) & 98 & Unclassified \\
\hline OTU57 & $1.0^{\mathrm{b}}$ & $0.0^{\mathrm{a}}$ & $0.0^{\mathrm{a}}$ & $0.0^{\mathrm{a}}$ & $0.0^{\mathrm{a}}$ & Uncultured bacterium (DQ125520.1) & 98 & Unclassified \\
\hline OTU58 & $1.0^{\mathrm{b}}$ & $0.0^{\mathrm{a}}$ & $4.5^{\mathrm{c}}$ & $11.0^{c}$ & $1.0^{\mathrm{b}}$ & Uncultured bacterium (DQ226081.1) & 99 & Unclassified \\
\hline OTU59 & $0.0^{\mathrm{a}}$ & $0.0^{\mathrm{a}}$ & $4.5^{\mathrm{b}}$ & $0.0^{\mathrm{a}}$ & $0.0^{\mathrm{a}}$ & Uncultured bacterium (DQ228365.1) & 94 & Unclassified \\
\hline OTU60 & $1.0^{\mathrm{b}}$ & $0.0^{\mathrm{a}}$ & $0.0^{\mathrm{a}}$ & $0.0^{\mathrm{a}}$ & $0.0^{\mathrm{a}}$ & Uncultured bacterium (DQ256349.1) & 99 & Unclassified \\
\hline OTU61 & $0.0^{\mathrm{a}}$ & $0.0^{\mathrm{a}}$ & $0.0^{\mathrm{a}}$ & $2.0^{\mathrm{b}}$ & $0.0^{\mathrm{a}}$ & Uncultured bacterium (DQ264533.1) & 99 & Unclassified \\
\hline OTU62 & $0.0^{\mathrm{a}}$ & $0.0^{\mathrm{a}}$ & $0.0^{\mathrm{a}}$ & $1.0^{\mathrm{b}}$ & $0.0^{\mathrm{a}}$ & Uncultured bacterium (DQ264605.1) & 99 & Unclassified \\
\hline OTU63 & $0.0^{\mathrm{a}}$ & $0.0^{\mathrm{a}}$ & $0.0^{\mathrm{a}}$ & $4.0^{\mathrm{b}}$ & $0.0^{\mathrm{a}}$ & Uncultured bacterium (DQ264645.1) & 99 & Unclassified \\
\hline OTU64 & $0.0^{\mathrm{a}}$ & $0.0^{\mathrm{a}}$ & $2.3^{\mathrm{b}}$ & $13.0^{c}$ & $0.0^{\mathrm{a}}$ & Uncultured bacterium (DQ394301.1) & 99 & Unclassified \\
\hline OTU65 & $0.0^{\mathrm{a}}$ & $0.0^{\mathrm{a}}$ & $0.0^{\mathrm{a}}$ & $0.0^{\mathrm{a}}$ & $2.0^{\mathrm{b}}$ & Uncultured bacterium (DQ415787.1) & 99 & Unclassified \\
\hline OTU66 & $2.0^{\mathrm{b}}$ & $0.0^{\mathrm{a}}$ & $0.0^{\mathrm{a}}$ & $0.0^{\mathrm{a}}$ & $0.0^{\mathrm{a}}$ & Uncultured bacterium (DQ455576.1) & 94 & Unclassified \\
\hline OTU67 & $1.0^{\mathrm{b}}$ & $0.0^{\mathrm{a}}$ & $2.3^{\mathrm{bc}}$ & $6.0^{\mathrm{c}}$ & $0.0^{\mathrm{a}}$ & Uncultured bacterium (DQ532284.1) & 98 & Unclassified \\
\hline OTU68 & $0.0^{\mathrm{a}}$ & $14.7^{\mathrm{b}}$ & $0.0^{\mathrm{a}}$ & $0.0^{\mathrm{a}}$ & $5.0^{\mathrm{b}}$ & Uncultured bacterium (DQ675075.1) & 99 & Unclassified \\
\hline OTU69 & $0.0^{\mathrm{a}}$ & $0.0^{\mathrm{a}}$ & $0.0^{\mathrm{a}}$ & $2.0^{\mathrm{b}}$ & $0.0^{\mathrm{a}}$ & Uncultured bacterium (EF632913.1) & 99 & Unclassified \\
\hline OTU70 & $0.0^{\mathrm{a}}$ & $16.7^{\mathrm{c}}$ & $0.0^{\mathrm{a}}$ & $1.0^{\mathrm{b}}$ & $2.0^{\mathrm{b}}$ & Uncultured bacterium (EF655641.1) & 99 & Unclassified \\
\hline OTU71 & $0.0^{\mathrm{a}}$ & $0.0^{\mathrm{a}}$ & $0.0^{\mathrm{a}}$ & $1.0^{\mathrm{b}}$ & $0.0^{\mathrm{a}}$ & Uncultured bacterium (EF999404.1) & 99 & Unclassified \\
\hline OTU72 & $1.0^{\mathrm{b}}$ & $0.0^{\mathrm{a}}$ & $0.0^{\mathrm{a}}$ & $0.0^{\mathrm{a}}$ & $0.0^{\mathrm{a}}$ & Uncultured bacterium (EU024330.1) & 99 & Unclassified \\
\hline OTU73 & $1.0^{\mathrm{b}}$ & $0.0^{\mathrm{a}}$ & $0.0^{\mathrm{a}}$ & $0.0^{\mathrm{a}}$ & $0.0^{\mathrm{a}}$ & Uncultured bacterium (EU234087.1) & 95 & Unclassified \\
\hline OTU74 & $6.0^{\mathrm{b}}$ & $0.0^{\mathrm{a}}$ & $0.0^{\mathrm{a}}$ & $0.0^{\mathrm{a}}$ & $0.0^{\mathrm{a}}$ & Uncultured bacterium (EU358726.1) & 99 & Unclassified \\
\hline OTU75 & $1.0^{\mathrm{b}}$ & $0.0^{\mathrm{a}}$ & $0.0^{\mathrm{a}}$ & $0.0^{\mathrm{a}}$ & $0.0^{\mathrm{a}}$ & Uncultured bacterium (EU799211.1) & 99 & Unclassified \\
\hline OTU76 & $6.0^{\mathrm{b}}$ & $0.0^{\mathrm{a}}$ & $0.0^{\mathrm{a}}$ & $0.0^{\mathrm{a}}$ & $0.0^{\mathrm{a}}$ & Uncultured bacterium (FJ172868.1) & 91 & Unclassified \\
\hline
\end{tabular}

${ }^{*}$ Within each row, data marked with the same superscript reflect values within a $0.5-1.5$-fold difference range. OTU, operational taxonomic unit, the clones with $100 \%$ sequence similarity.

$\left(C_{\text {good }}\right)$ values, indicating that the microbiota in the gut content of grass carp was more diverse than in samples from the associated microbial environments. Rarefaction curves generated for the 16S rDNA clone libraries confirmed that the bacterial diversity of the grass carp gut content was greater than in associated environmental samples (Fig. 3).

The bacterial communities in samples from the associated environment were significantly different $\left(C_{\mathrm{s}}<0.50\right)$ from those in the gut content of grass carp
(Table 2). Nonetheless, the $C_{\mathrm{s}}$ values between samples from ingested food (feed or reed) and gut content $\left(C_{\mathrm{s}}=0.46\right.$ or 0.49 respectively) were higher than between samples from habitat (pond water or sediment) and gut content $\left(C_{\mathrm{s}}=0.28\right.$ or 0.34 respectively), indicating that the allochthonous intestinal microbiota of the grass carp was relatively closer to ingested food than to the habitat (Table 2).

The relative abundance of 25 OTUs $(1,3-6,9,11-$ $14,17-18,21,23-24,29,31,57,60,66$ and $72-76)$ in 


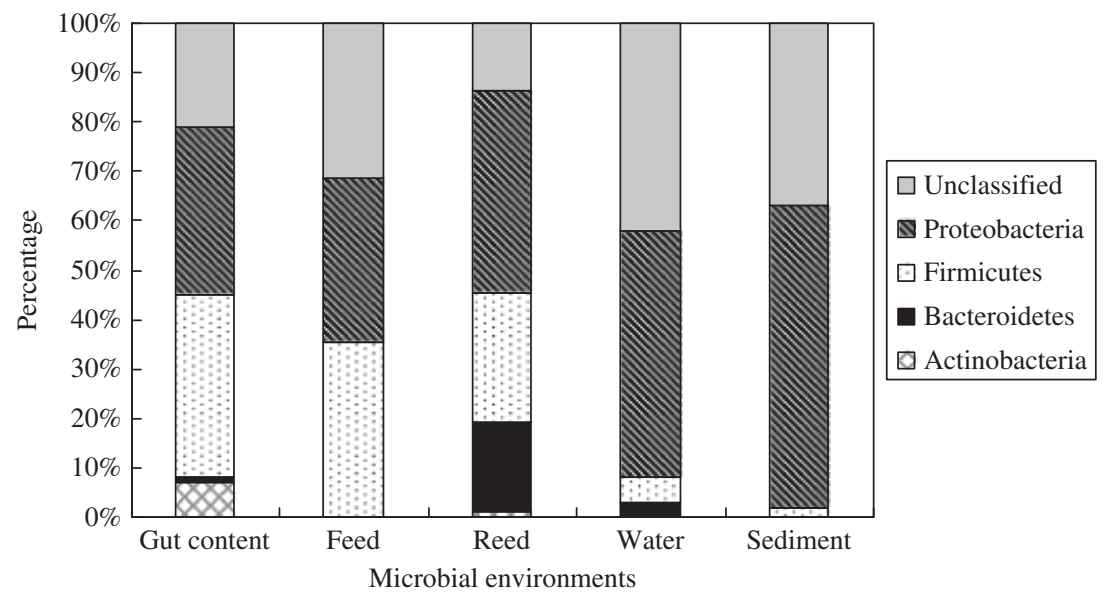

Figure 1 Bacterial phylum composition in the gut content of grass carp and in food and habitat samples. The bacterial phylum composition was calculated based on Table 1.
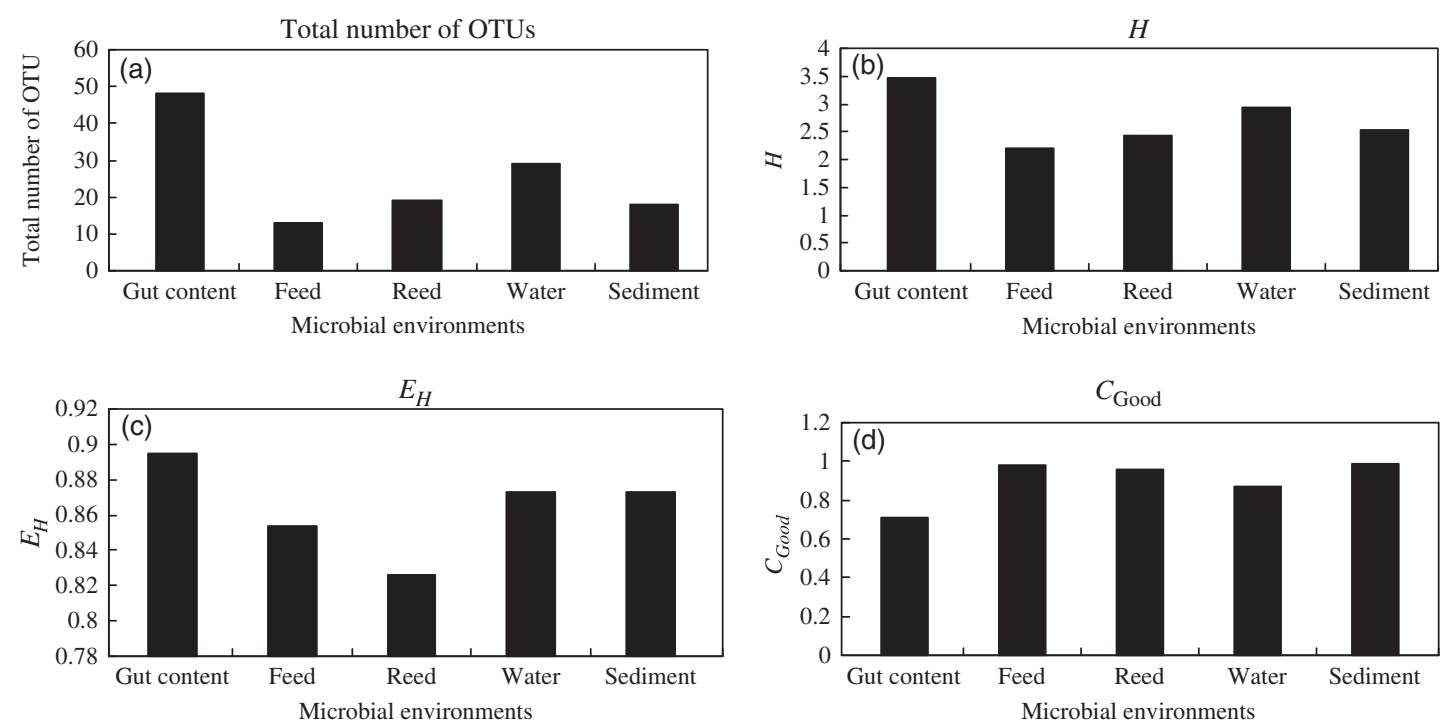

Figure 2 Bacterial diversity in the grass carp intestine and in the associated habitat and food samples (a) total number of operational taxonomic units (OTUs); (b) $H$; (c) $E_{H}$; (d) $C_{\text {good. }} H=-\Sigma R A_{i} \ln \left(R A_{i}\right) ; E_{H}=H / \ln (S)$ (where $R A_{i}$ is the proportion of the ith OTU and $S$ is the total number of OTUs) (Dethlefsen et al. 2008); $C_{\text {good }}=1-N_{1} /$ total number of OTUs (where $N_{1}$ is the number of OTUs with only one clone) (Good 1953).

gut content samples was substantially higher than that in samples from the associated environments (Table 1). Twenty-eight OTUs $(27,32,33,40-43,45$, $47-56,59,61-65$ and $68-71$ ) were identified in the associated environment samples. These OTUs were not detected in the digesta samples. Of the eight OTUs with a relative abundance of $\geq 3 \%$ in the gut content samples, six were identified in both gut content and its corresponding environment samples (OTUs 8, $22,34-36$ and 39) and two were identified only in the gut content samples (OTUs 74 and 76).

\section{Discussion}

To our knowledge, 16S rDNA clone library has been used in four studies to evaluate the intestinal microbial diversity in fish (Kim et al. 2007; Navarrete et al. 2009; Ward et al. 2009; the current study). In the present study, we used a universal primer set to construct $16 \mathrm{~S}$ rDNA gene libraries for identification of the allochthonous gut microbiota of grass carp and of the habitat and food samples. Many researchers have suggested that methods based on 16S rDNA 


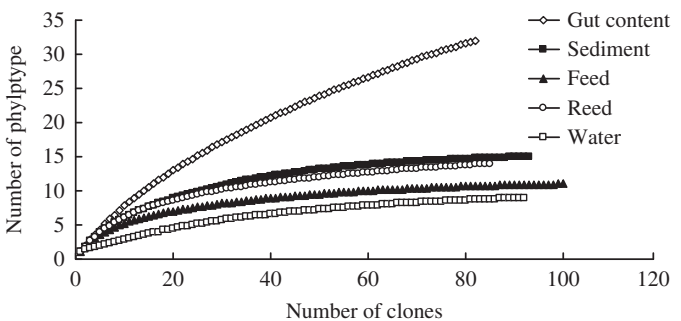

Figure 3 Rarefaction curves from 16S rDNA clone libraries from the gut content of grass carp and from associated habitat and food samples. Rarefaction curves were created using the species diversity function of the ЕсоSIM 700 statistical software (Gotelli \& Entsminger 2002).

Table 2 Pairwise similarity coefficients $\left(C_{s}\right)$ matrix for bacterial communities identified in the gut content of grass carp and in food and habitat samples*

\begin{tabular}{llllll}
\hline & Gut content & Feed & Reed & Water & Sediment \\
\hline Gut content & 1.00 & & & & \\
Feed & $0.46^{* *}$ & 1.00 & & & \\
Reed & $0.49^{* *}$ & $0.71^{\dagger}$ & 1.00 & & \\
Water & $0.28^{* *}$ & $0.58^{* *}$ & $0.68^{\dagger}$ & 1.00 & \\
Sediment & $0.34^{* *}$ & $0.75^{\dagger}$ & $0.67^{\dagger}$ & $0.57^{* *}$ & 1.00 \\
\hline
\end{tabular}

*In this study, $C_{s}<0.60$ is regarded as a significant difference; that of $0.60 \leq C_{s}<0.80$ is a marginal difference; and that $\geq 0.80$ is very similar.

** Significantly different.

${ }^{\dagger}$ Marginally different.

gene sequences using universal primers may not accurately reflect the true underlying diversity of a given environment (Marchesi, Sato, Weightman, Martin, Fry, Hiom \& Wade 1998; Suzuki \& Giovannoni 1996). In addition, technical challenges such as PCR bias, varying ribosomal DNA copy numbers and the efficiency of DNA extraction procedures all have the potential to significantly skew abundance estimates; therefore, assumption of a direct relationship between the number of sequences of a particular type in a clone library and the number of organisms in the environment may be inaccurate (Marchesi et al. 1998; Suzuki \& Giovannoni 1996). However, generation of a 16S rDNA clone library using sequences that are almost full length improves the accuracy of species identification (Brons \& Elsas 2008). The current study used different methods to extract DNA from different sample types, which allowed for the better recovery of DNA (He, Zhou, Yao \& Bai 2009) and, subsequently, for identical PCR amplification reaction conditions. Thus, bias towards any individual sample would be similar across all sample types and would consequently be minimal when comparing the relative abundance of a specific OTU (Zhou, Liu, He, Shi, Gao, Yao \& Ringø 2009). Furthermore, relative abundance differences were considered to be significant only when the relative OTU abundance in any one sample type was $\geq 1.5$-fold higher or $\leq 0.5$-fold lower than that in any other sample type.

In the present study, Proteobacteria, Firmicutes and Actinobacteria were the dominant allochthonous microbiota in the gut content of grass carp cultured in pond, while Huang et al. (2009) reported three bacterial phyla, Proteobacteria, Firmicutes and Cyanobacteria, in the gut digesta of grass carp when the 16S rDNA V3 DGGE method was used. In previous studies using classic cultivation, Luo et al. (2001) identified Proteobacteria, Firmicutes, Bacteroides and Actinobacteria as the dominant allochthonous bacteria in the intestine of grass carp fed a commercial feed containing diverse components and nutrients, while Zhou et al. (1998) reported Proteobacteria, Bacteroides and Firmicutes in the gut content of grass carp fed either a commercial feed or Spirodela polyrhiza. Although different food types obviously change the bacterial composition of the gut (Zhou et al. 1998), the gut studies of grass carp indicate that Proteobacteria and Firmicutes comprise the microbiota phylogenetic core (the common phyla).

In previous investigations, it has been proposed that water and food are the sources of some of the bacteria present in the GI tract of fish (Verschuere, Rombaut, Sorgeloos \& Verstraete 2000; Olafsen 2001; Romero \& Navarrete 2006). Similar findings were observed in the present study; $75 \%$ of the OTUs with a relative abundance $\geq 3 \%$ in the gut content were identified in feed and habitat samples. However, we observed that the similarity coefficients between gut microbiota and microbiota from the associated environment were quite low $\left(C_{\mathrm{s}}<0.50\right)$, indicating that a substantial number of grass carp gut microbiota are distinct from the corresponding environmental microbiota. Furthermore, the gut content of grass carp raised in the pond showed the highest bacterial diversity compared with its surrounding environments, supporting by the total number of OTUs, Shannon diversity index $(H)$, Shannon equitability index $\left(E_{H}\right)$, Coverage $\left(C_{\text {good }}\right)$ and rarefaction curves, which might reflect the uniqueness of the host gut environment. In addition, the $C_{\text {good }}$ values observed in all the samples were larger than 0.70 , which indicates that the clone number analysed in each sample 
in the present study is accepted as valid in microbial diversity analysis (Pace 1997).

$\beta$-Proteobacteria have been reported to predominate in freshwater and freshwater sediment (Bissett, Bowman \& Burke 2006). However, in the present study, $\gamma$-Proteobacteria were the most abundant bacteria in all samples, except for reed samples (Bacteroidetes, still not $\beta$-Proteobacteria). As $\gamma$-Proteobacteria are usually found in oligotrophic environments such as marine sediments and seawaters (Grey \& Herwig 1996; Urakawa, Kita-Tsukamoto \& Ohwada 1999; Bowman \& McCuaig 2003; Kawahara, Nishi, Hisano, Fukui, Yamaguchi \& Mochizuki 2009), we suggest that the sampling pond was nutrient deficient. Actually, this pond was recently converted from a natural reed pond to an artificial feed-based rearing pond for poly-cultured fish species including grass carp (Wang et al. 2010).

Westerdahl, Olsson, Kjelleberg and Conway (1991) suggested that all fish had indigenous bacteria with inhibitory effects in protecting the host against pathogens. Probiotics are thought to be beneficial for the host by improving the intestinal microbial balance via inhibition of pathogens and toxin-producing bacteria (Lilly \& Stillwell 1965; Fuller 1989; Irianto \& Austin 2002). Therefore, the fish intestinal microbiota might be a key pool of potential probiotics for cultured fish species. Bacillus spp. (B. megaterium, B. polymyxa, B. subtilis, B. lichenifomis), lactic acid bacteria (Lactobacillus spp., Carnobacterium spp., Streptococcus spp.), Pseudomonas sp. (P. fluorescens) and Vibrio sp. (V. alginolyticus, V. salmonicida like) have been examined as probiotics for aquaculture (Gatesoupe 1999; Verschuere et al. 2000). In the present study, several potential probiotic strains of Bacillus spp. were detected: B. coagulans (OTU6, identity $99 \%$ ), which has been reported to have the ability to ferment biomass-derived sugars to lactic acid (Patel, Ou, Harbrucker, Aldrich, Buszko, Ingram \& Shanmugam 2006); B. massiliensis (OTU7, identity 99\%), having thermostable hydantoinase and carbamoylase activity (Mei, He, Liu \& Ouyang 2009); and B. megaterium (OTU8, identity 99\%), which has been recommended as a probiotic in aquaculture by Gatesoupe (1999). Furthermore, B. pumilus (OTU9, identity 99\%), isolated firstly from penaeid shrimp (Penaeus monodon) and found to be inhibitory against marine pathogens such as $V$. alginolyticus, $V$. mimicus and V. harveyi (Hill, Baiano \& Barnes 2009), was also identified in the gut digesta of grass carp.

Lactobacillus curvatus-like, Lactobacillus fermentum-like, Streptococcus sp.-like (Gatesoupe 1999;
Verschuere et al. 2000) and Lactococcus lactis-like (Itoi, Yuasa, Washio, Abe, Ikuno \& Sugita 2009) bacteria, which have been suggested previously to be potential probiotic candidates in aquaculture, were also identified from the gut content of grass carp. Although one or several probiotic characterizations of these bacteria were suggested in the concerned studies, further studies are required to clarify whether these bacteria are suitable as probiotics to cultured grass carp. Streptococcus iniae-like bacterium, previously isolated from diseased fish and identified as fish pathogen (Bachrach, Zlotkin, Hurvitz, Evans \& Eldar 2001), was identified in the gut digesta of grass carp. Based on our results, this bacterium might originate from the pond water. To clarify this hypothesis, additional studies are necessary.

\section{Acknowledgments}

This work was supported by the National Natural Science Foundation of China (30972265) and the Key Program of Transgenic Plant Breeding (2008ZX08011005; 2009ZX08012-024B).

\section{References}

Altschul S.F., Gish W., Miller W., Myers E.W. \& Lipman D.J. (1990) Basic local alignment search tool. Journal of Molecular Biology 215, 403-410.

Bachrach G., Zlotkin A., Hurvitz A., Evans D.L. \& Eldar A. (2001) Recovery of Streptococcus iniae from diseased fish previously vaccinated with a Streptococcus vaccine. Applied and Environmental Microbiology 67, 3756-3758.

Bissett A., Bowman J. \& Burke C. (2006) Bacterial diversity in organically-enriched fish farm sediments. FEMS Microbiology Ecology 55, 48-56.

Bowman J.P. \& McCuaig R.D. (2003) Biodiversity, community structural shifts, and biogeography of prokaryotes within Antarctic continental shelf sediment. Applied and Environmental Microbiology 69, 2463-2483.

Brons J.K. \& Elsas J.D. (2008) Analysis of bacterial communities in soil by denaturing gradient gel electrophoresis and clone libraries as influenced by different reverse primers. Applied and Environmental Microbiology $\mathbf{7 4 ,}$ 2717-2727.

Chilton E.W. \& Muoneke M.I. (1983) Biology and management of grass carp (Ctenopharyngodon idella, Cyprinidae) for vegetation control: a North American perspective. Reviews in Fish Biology and Fisheries 2, 283-320.

Cole J.R., Chai B., Farris R.J., Wang Q., Julam S.A., McGarrel D.M., Garrity G.M. \& Tiedje J.M. (2005) The ribosomal database project (RDP-II): sequences and tools for 
high-throughput rRNA analysis. Nucleic Acids Research 33, D294-D296.

Dethlefsen L., Huse S., Sogin M.L. \& Relman D.A. (2008) The pervasive effects of an antibiotic on the human gut microbiota, as revealed by deep $16 \mathrm{~S}$ rRNA sequencing. PLoS Biology 6, 2383-2400.

Fuller R. (1989) Probiotics in man and animals. Journal of Applied Bacteriology 66, 365-378.

Gatesoupe F.J. (1999) The use of probiotics in aquaculture. Aquaculture 180, 147-165.

Gernert C., Glöckner F.O., Krohne G. \& Hentschel U. (2005) Microbial diversity of the freshwater sponge Spongilla lacustris. Microbiology Ecology 50, 206-212.

Gómez G.D. \& Balcázar J.L. (2008) A review on the interactions between gut microbiota and innate immunity of fish. FEMS Immunology and Medical Microbiology 52 , 145-154.

Good I.L. (1953) The population frequencies of species and estimation of population parameters. Biometrika 40, 237-264.

Gotelli N.J. \& Entsminger G.L. (2002) EcoSim: Null Models Software for Ecology, Version 7. Acquired Intelligence and Kesey-Bear, Jericho,VT, USA. Available at http://homepages. together.net/ gentsmin/ecosim.htm.

Grey J.P. \& Herwig R.P. (1996) Phylogenetic analysis of the bacterial communities in marine sediments. Applied and Environmental Microbiology 62, 4049-4059.

He L., Zhang Z., Xie C., Hao B., Wang C. \& He G. (2008) Isolation of cellulose-producing microbes from the intestine of grass carp (Ctenopharyngodon idellus). Environmental Biology of Fishes 272, 140-145.

He S., Zhou Z., Liu Y., Shi P., Yao B., Ringø E. \& Yoon I. (2009) Effects dietary Saccharomyces cerevisiae fermentation product (DVAQUA ${ }^{\text {T. }}$ ) on growth performance, intestinal autochthonous bacterial community and non-specific immunity of hybrid tilapia (Oreochromis niloticus $+\times 0$.

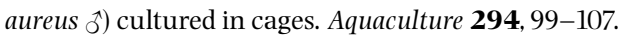

He S., Zhou Z., Yao B. \& Bai D. (2009) Effects of three different DNA extraction methods on the analysis of bacteria community from different micro-ecological environments in a farming pond by PCR-DGGE. Journal of Agricultural Science and Technology 11, 73-79 (article in Chinese with English abstract).

Hill J.E., Baiano J.C. \& Barnes A.C. (2009) Isolation of a novel strain of Bacillus pumilus from penaeid shrimp that is inhibitory against marine pathogens. Journal of Fish Diseases 32, 1007-1016.

Hooper L.V., Midtvedt T. \& Gordon J.I. (2002) How hostmicrobial interactions shape the nutrient environment of the mammalian intestine. Annual Review Nutrition 22, 283-307.

Huang H., Shi P., Wang Y., Luo H., Shao N., Wang G., Yang P. \& Yao B. (2009) Gene diversity of beta-propeller phytase in the intestinal contents of grass carp insight into the major phosphorus release from phytate in nature. Applied and Environmental Microbiology 75, 1508-1516.
Huber I., Spanggaard B., Appel K.F., Rossen L., Nielsen T. \& Gram L. (2004) Phylogenetic analysis and in situ identification of the intestinal microbial community of rainbow trout (Oncorhynchus mykiss, Walbaum). Journal of Applied Microbiology 96, 117-132.

Irianto A. \& Austin B. (2002) Probiotics in aquaculture. Journal of Fish Diseases 25, 633-642.

Itoi S., Yuasa K., Washio S., Abe T., Ikuno E. \& Sugita H. (2009) Phenotypic variation in Lactococcus lactis subsp. lactis isolates derived from intestinal tracts of marine and freshwater fish. Journal of Applied Microbiology 107, 867-874.

Kawahara A., Nishi T., Hisano Y., Fukui H., Yamaguchi A. \& Mochizuki N. (2009) The sphingolipid transporter spns functions in migration of zebrafish myocardial precursors. Science 323, 524-527.

Kim D.-H., Brunt J. \& Austin B. (2007) Microbial diversity of intestinal contents and mucus in rainbow trout (Oncorhynchus mykiss). Journal of Applied Microbiology 102, 1654-1664.

Lilly D.M. \& Stillwell R.H. (1965) Probiotics growth promoting factors produced by micro-organisms. Science 147, $747-748$.

Luo L., Chen X. \& Cai X. (2001) Effects of Andrographis paniculata on the variation of intestinal microflora of Ctenopharyngodon idellus. Journal of Fisheries of China $\mathbf{2 5}$, 232-237. (article in Chinese with English abstract).

Macfarlane G.T. \& Macfarlane S. (1997) Human colonic microbiota: ecology, physiology and metabolic potential of intestinal bacteria. Scandinavian Journal of Gastroenterology 32 (Suppl. 222), 3-9.

Marchesi J.R., Sato T., Weightman A.J., Martin T.A., Fry J.C., Hiom S.J. \& Wade W.G. (1998) Design and evaluation of useful bacterium-specific PCR primers that amplify genes coding for bacterial $16 \mathrm{~S}$ rRNA. Applied and Environmental Microbiology 64, 795-799.

Martin-Laurent F., Philippot L., Hallet S., Chaussod R., Germon J.C., Soulas G. \& Catroux G. (2001) DNA extraction from soils: old bias for new microbial diversity analysis methods. Applied and Environmental Microbiology 67, 2354-2359.

Mei Y., He B., Liu N. \& Ouyang P. (2009) Screening and distributing features of bacteria with hydantoinase and carbamoylase. Microbiology Research 164, 322-329.

Ministry of Agriculture, China (2009) China Fishery Products Annual 2008. China Agriculture Press, Beijing, China.

Muyzer G., Waal E.C. \& Uitterlinden A.G. (1993) Profiling of complex microbial populations by denaturing gradient gel electrophoresis analysis of polymerase chain reaction-amplified genes coding for 16S rRNA. Applied and Environmental Microbiology 59, 695-700.

Myers R.M., Fischer S.G., Lerman L.S. \& Maniatis T. (1985) Nearly all single base substitutions in DNA fragments joined to a GC-clamp can be detected by denaturing gradient gel electrophoresis. Nucleic Acids Research 13, 3131-3145. 
Navarrete P., Espejo R.T. \& Romero J. (2009) Molecular analysis of microbiota along the digestive tract of juvenile Atlantic salmon (Salmo salar L.). Microbial Ecology 57, 550-561.

Navarrete P., Magne F., Mardones P., Riveros M., Opazo R., Suau A., Pochart P. \& Romero J. (2010) Molecular analysis of intestinal microbiota of rainbow trout (Oncorhynchus mykiss). FEMS Microbiology Ecology 71, 148-156.

Nieto T.P., Toranzo A.E. \& Barja J.L. (1984) Comparison between the bacterial flora associated with fingerling rainbow trout cultured in two different hatcheries in the north-west of Spain. Aquaculture 42, 193-206.

Olafsen J. (2001) Interactions between fish larvae and bacteria in marine aquaculture. Aquaculture 200, 223-247.

Pace N.R. (1997) A molecular view of microbial diversity and the biosphere. Science 276, 734-740.

Patel M.A., Ou M.S., Harbrucker R., Aldrich H.C., Buszko M.L., Ingram L.O. \& Shanmugam K.T. (2006) Isolation and characterization of acid-tolerant, thermophilic bacteria for effective fermentation of biomass-derived sugars to lactic acid. Applied and Environmental Microbiology $\mathbf{7 2}$, 3228-3235.

Romero J. \& Navarrete P. (2006) 16S rDNA-Based analysis of dominant bacterial populations associated with early life stages of coho salmon (Oncorhynchus kisutch). Microbial Ecology 51, 422-430.

Spanggaard B., Huber I., Nielsen J., Nielsen T., Appel K.F. \& Gram L. (2000) The microflora of rainbow trout intestine: a comparison of traditional and molecular identification. Aquaculture 182, 1-15.

Sugita H., Oshima K.,Tamura M. \& Deguchi Y. (1983) Bacterial flora in the gastrointestine of freshwater fishes in the river. Bulletin of the Japanese Society of Scientific Fisheries 49, 1387-1395.

Suzuki B. \& Giovannoni S.J. (1996) Bias caused by template annealing in the amplification of mixtures of 16S rRNA genes by PCR. Applied and Environmental Microbiology 62, 625-630.

Thakuria D., Schmidt O., Mac Siúrtáin M., Egan D. \& Doohan F.M. (2008) Importance of DNA quality in comparative soil microbial community structure analyses. Soil Biology and Biochemistry 40, 1390-1403.
Trust T.J., Bull L.M., Currie B.R. \& Buckley J.T. (1979) Obligate anaerobic bacteria in the gastrointestinal microflora of the grass carp (Ctenopharyngodon idella), goldfish (Carassius auratus), and rainbow trout (Salmo gairdneri). Journal of the Fisheries Research Board of Canada 36, $1174-1179$.

Tsai Y.L. \& Olson B.H. (1991) Rapid method for direct extraction of DNA from soil and sediments. Applied and Environmental Microbiology 57, 1070-1074.

Urakawa H., Kita-Tsukamoto K. \& Ohwada K. (1999) Microbial diversity on marinesediments from Sagami Bay and Tokyo Bay, Japan, as determined by 16S rRNA gene analysis. Microbiology 145, 3305-3315.

Verschuere L., Rombaut G., Sorgeloos P. \& Verstraete W. (2000) Probiotic bacteria as biological control agents in aquaculture. Microbiology and Molecular Biology Reviews 64, 655-671.

Wang W., Zhou Z., He S., Liu Y., Cao Y., Shi P., Yao B. \& Ring $\varnothing$ E. (2010) Identification of the adherent microbiota on gills and skins of poly-cultured gibel carp (Carassius auratus gibelio) and bluntnose black bream (Megalorama mablycephala Yih). Aquaculture Research, doi: 10.1111/j.13652109.2009.0259.x.

Ward N., Blaire S., Penn K., Methé B. \& Detrich H.W. (2009) Characterization of the intestinal microbiota of two Antarctic Notothenioid fish species. Extremophiles 13, 679-685.

Westerdahl A., Olsson J.C., Kjelleberg S. \& Conway P.L. (1991) Isolation and characterization of turbot (Scophtalmus maximus)-associated bacteria with inhibitory effects against Vibrio anguillarum. Applied and Environmental Microbiology 57, 2223-2228.

Zhou W., Chen X., Zhang D. \& Chen C. (1998) A preliminary study on the influence of different feeding stuff on intestinal microflora of grass carp (Ctenopharyngodon idellus). Journal of Huazhong Agricultural University 17, 252-256. (article in Chinese with English abstract).

Zhou Z., Liu Y., He S., Shi P., Gao X., Yao B. \& Ringø E. (2009) Effects of dietary potassium diformate (KDF) on growth performance, feed conversion and intestinal bacterial community of hybrid tilapia (Oreochromis niloticus ㅇ $\times$ O. aureus 3 ) $)$. Aquaculture 291, 89-94. 